\title{
ABUNDANCIA FLORÍSTICA DE LA QUEBRADA LA GRANDE EN LA VEREDA CENTRO TIBASOSA-BOYACÁ
}

\author{
FLORISTIC ABUNDANCE OF LA GRANDE STREAM IN THE VEREDA CENTRO \\ TIBASOSA-BOYACÁ
}

Luisa Fernanda Rubiano

Estudiante programa Tecnología en Sistemas Agroforestales -Universidad

Nacional Abierta y a Distancia

https://orcid.org/0000-0003-1587-896X

Irubianol@unadvirtual.edu.co

Yenny Maritza Camacho Torres

Docente -Universidad Nacional Abierta y a Distancia

https://orcid.org/0000-0001-8668-6274

yenny.camacho@unad.edu.co

\section{RESUMEN}

Contextualización: El municipio de Tibasosa y en especial la quebrada la Grande se caracteriza por poseer ecosistemas estratégicos que debido a su ubicación geográfica y sus condiciones edafoclimáticas presentan ciertas problemáticas ligadas a la explotación agrícola y pecuaria representada en monocultivos y ganadería extensiva

Vacío de conocimiento: A pesar del fuerte impacto ocasionado por la actividad no se observa la implementación de medidas de evaluación ni mucho menos de control frente a los continuos cambios producto de dichas actividades.

Propósito: obtener información real de la zona basada en el reconocimiento de la composición florística de la parte media de la quebrada la Grande en la vereda Centro en el Municipio de Tibasosa, información relevante para la implementación de medidas de mitigación aplicadas a la quebrada considerada como uno de los afluentes más importantes por considerarse como un abastecedor del recurso hídrico para el acueducto de la ciudad.

DOI: https://doi.org/10.22490/26653176.4319 
Metodología: se seleccionó un área de muestreo de 2,63 hectáreas donde se desarrolló un recorrido exploratorio y se identificaron las especies más representativas.

Resultados y conclusiones: se identificaron 183 individuos, representados en 15 familias y 17 géneros los cuales ofrecieron diversos usos; entre los que se cuentan la protección de ríos, aprovechamiento de productos maderables no maderables y medicinales, a partir de los cuales se propusieron medidas de conservación como el desarrollo de proyectos de producción sostenible o el establecimiento de sistemas agroforestales como una alternativa frente a la degradación ambiental presente en la zona como consecuencia de las actividades de agricultura intensiva adelantadas en la misma.

Palabras clave: Microcuenca abastecedora, conservación, Tibasosa

\section{ABSTRACT}

Contextualization: The municipality of Tibasosa and especially the Quebrada la Grande is characterized by having strategic ecosystems that, due to their geographical location and their edaphoclimatic conditions, present certain problems linked to the agricultural and livestock exploitation represented in monocultures and extensive cattle ranching.

Knowledge gap: Despite the strong impact caused by the activity, the implementation of evaluation measures, much less control measures, is not observed in the face of the continuous changes resulting from said activities.

Purpose: obtain real information on the area based on the recognition of the floristic composition of the middle part of the La Grande stream in the Centro village in the Municipality of Tibasosa, relevant information for the implementation of mitigation measures applied to the stream considered one of the the most important tributaries for being considered as a supplier of the water resource for the aqueduct of the city.

Methodology: A 2.63-hectare sampling area was selected where an exploratory tour was developed and the most representative species were identified.

Results and conclusions: 183 individuals were identified, represented in 15 families and 17 genera which offered various uses; Among which are the protection of rivers, use of nontimber and medicinal timber products, from which conservation measures such as the development of sustainable production projects or the establishment of agroforestry systems as an alternative to degradation were proposed. environmental present in the area as a consequence of the intensive agriculture activities carried out in it.

Keywords: Micro-basin supplying, conservation, Tibasosa 


\section{RESUMEN GRÁFICO}

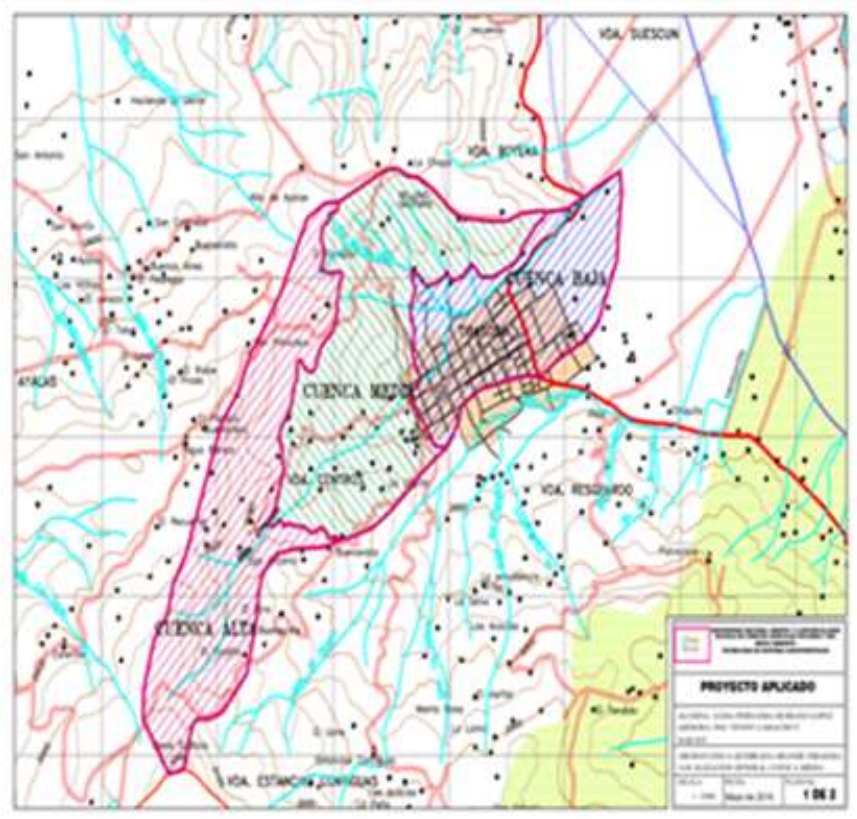

Rubiano, L. (2019) Delimitación de la Microcuenca. Sectorización de la quebrada la Grande (partes Alta, Media y Baja).

\section{INTRODUCCIÓN}

Colombia posee una biodiversidad reconocida a nivel mundial, ocupa el primer lugar en especies de aves y orquídeas, el segundo en el mundo en riqueza de plantas, anfibios, mariposas y peces de agua dulce y el cuarto lugar en mamíferos (Humboldt ,2017). Esta condición de biodiversidad habla de la riqueza de ecosistemas que posee el país, debido a su posición geográfica y su clima haciendo que el patrimonio natural se vea reflejado en todas las latitudes. Es así como empezamos por referenciar a los bosques alto andinos cuyos ecosistemas albergan importantes muestras de biodiversidad, constituyéndose en el habitad de numerosas especies que tiene ente sus funciones la regulación hídrica de la zonas (Abud y Torres, 2016). Estas características climáticas y fisiográficas influyen en la fauna y flora de cada región, otorgando especies vegetales que caracterizan a ecosistemas estratégicos. En virtud de esto y como una necesidad para mantener las cualidades de dichos ecosistemas se plantea la posibilidad de identificar y reconocer las principales especies vegetales ubicadas en la parcela de estudio de la quebrada la grande del municipio de Tibasosa.

El municipio de Tibasosa y en especial la quebrada la grande se han visto afectados por las actividades productivas derivadas de la explotación agrícola y pecuaria, representada en monocultivos y ganadería extensiva que llevan a la sustitución de especies nativas por 
cultivos agrícolas de pan coger y pasturas, provocando la extinción y desplazamiento de especies propias de la zona, situación que se arraiga, debido a la cercanía de la cuenca con el centro poblado que junto con la disponibilidad de agua hacen de este lugar óptimo para la explotación agrícola y pecuaria a pequeña escala (Tibasosa, 2019).

De acuerdo a lo anterior se propone la identificación florística como una herramienta para conocer la vegetación predominante de tal forma que a partir del reconocimiento de dichas especies se puedan plantear estrategias de manejo sustentables con las necesidades de la cuenca pues como lo menciona Romero (2000) las actividades de los sistemas intensivos como la agricultura, modifican los microorganismos de los ecosistemas naturales, que resulta en una alteración directa a la flora previa de la zona.

De acuerdo a esto se planteó como objetivo general, realizar la identificación de la abundancia florística de la cuenca media de la quebrada La Grande, donde se identificarían las especies nativas y exóticas predominante en la zona. Para el cumplimiento de este propósito fue necesario el desarrollo de un recorrido exploratorio, una estrategia basada en un diagnostico preliminar a partir de visitas de campo, que permitió el posterior levantamiento clasificación taxonómica y aplicación de claves dendrologicas para el reconocimiento y cuantificación de especies arbustivas y forestales, que contribuyó con el registro de las especies encontradas en la zona, teniendo en cuenta que la realización de inventarios para cuantificar los recursos forestales es relativamente reciente y todavía no se ha generalizado su uso (Ledo et.al, 2012).

\section{MATERIALES Y MÉTODOS}

El trabajo de campo fue realizado durante los meses de abril a junio en la cuenca media de la quebrada Grande entre los 2550 y 2700 m de elevación.

La microcuenca de la quebrada Grande está ubicada en el municipio de Tibasosa el cual hace parte del Valle de Sogamoso. La Microcuenca la Grande nace en el cerro de Guatika, la parte media de la Microcuenca se encuentra entre las cotas, 2550-2700 m.s.n.m. según el EOT, se considera a esta microcuenca como la segunda en jerarquía, dentro del municipio, dada su importancia estratégica para el suministro de agua de uno de los acueductos Municipales, que hacen que esta sea considerada como fundamental para consumo humano y para irrigación de potreros.

Para poder obtener el inventario se empleó un muestreo no probabilístico exploratorio basado en observación directa en campo (Ferreira, 2018), toma de datos y registro de especies en la zona, todo aplicado a parcela temporal delimitada para el desarrollo del estudio, esto enmarcado en lo que menciona la FAO (2020) sobre la necesidad de seleccionar un subconjunto de una población que será sometida al muestreo en el marco de la obtención del inventario forestal, para esto fue necesario el desarrollo de un diagnóstico preliminar a partir de visitas de campo, la aplicación de técnicas de clasificación taxonómica y claves 
dendrologicas usadas en el reconocimiento, recolección y cuantificación de especies arbustivas y forestales encontradas en la zona.

Es así como a partir de la base topográfica obtenida del esquema de ordenamiento territorial (EOT) a escala 1:25.000 y con curvas de nivel cada 50 metros, se estableció el punto de interés sobre el cual se definió la delimitación exacta para posteriormente obtener un perfil topográfico del cauce de la quebrada y así llegar de manera precisa a la determinación exacta de la cuenta media motivo de estudio.

A partir de la delimitación exacta de la cuenca media se procedió al establecimiento de parcela temporal de observación dentro de un área de 2,63 hectáreas ubicadas todas en la vereda centro del municipio de Tibasosa. Es así como se propicia una demarcación y georreferenciación de sitios con características relevantes dentro del paisaje; identificando especies, alturas y diámetro a altura de pecho DAP, actividad desarrollada en estricto sentido de las manecillas del reloj a fin de evitar errores en el muestreo, hasta llegar nuevamente al punto de partida.

La identificación de especies se desarrolló a partir de la observación en campo de la parcela temporal, donde se registró nombre común o familia; es importante tener en cuenta que por ser especies características de la zona de vida de bosque seco montano bajo (bsMB) según Holdridge, de acuerdo a lo reportado en el esquema de ordenamiento territorial (EOT, 2016) estas se encontraron en diferentes estados de maduración. Lo que permitió identificar diferencias morfológicas de acuerdo a su desarrollo y así garantizar una adecuada clasificación. A partir del nombre común y familia se realizó la consulta a través de guías botánicas hasta llegar al nombre científico de cada especie encontrada. Para el caso de árboles con alturas superiores a $4 \mathrm{~m}$ y DAP mayor a $10 \mathrm{~cm}$, se someten a tabulación para determinar especies dominantes y/o con más tiempo en el área, determinando así si son nativas o introducidas. Para las no reconocidas en campo fue necesario realizar colecta y toma de fotografías para su posterior consulta a través de clave dicotómica (tipo de hojas, disposición, flores y/o frutos), mediante esta técnica se logró confirmar familia y género, indispensable en la determinación de la composición florística de la zona y diversidad de la misma.

\section{RESULTADOS Y DISCUSIÓN}

A partir de los datos obtenidos en la parcela de muestreo de la parte media de la microcuenca fue posible corroborar las especies identificadas mediante libros y herbarios online (Mahecha, 2004; Herbario Universidad de Antioquia,2008; Catálogo virtual de flora del Valle de Aburrá 2019 ), registrándose 183 individuos, representados en 15 familias y 17 especies, como se observa en la tabla 1 , donde hay una diversidad de especies, pero no hay una familia representativa, de acuerdo con los parámetros que permiten medir diversidad. 
Tabla 1. Especies encontradas en parcela de estudio

\begin{tabular}{cllll}
\hline NO. & FAMILIA & NOMBRE CIENTÍfICO & NOMBRE COMÚN & $\begin{array}{l}\text { NÚMERO DE } \\
\text { INDIVIDUOS }\end{array}$ \\
\hline 1 & SALICAEAE & Salix babylonica & Sauce llorón & 6 \\
2 & ADOXÁCEAE & Sambucus nigra & Sauco & 5 \\
3 & MYRTACEAE & Eugenia myrtifolia & Eugenia & 5 \\
4 & FABACEAE & Acacia melanoxylon & Acacia & 10 \\
5 & MORÁCEAE & Ficus carica & Higuera & 9 \\
6 & MYRSINACEAE & Myrsine guianensis & Cucharo & 21 \\
7 & SAPINDACEAE & Dodonaea viscosa & Hayuelo & 10 \\
8 & ERICACEAE & Vaccinium meridionale & Mortiño & 15 \\
9 & EUPHORBIACEAE & Ricinus communis & Higuerilla & 8 \\
10 & FLACOURTIACEAE & Xilosma spiculeferum & Corono,tabe & 12 \\
11 & EUPHORBIACEAE & Croton sp & Drago & 16 \\
12 & BETULIACEAE & Alnus acuminata & Aliso & 15 \\
13 & COMPOSITAE & Baccharis macranta & Ciro-camiseto & 9 \\
14 & COMPOSITAE & Baccharis latifolia & Chilco & 10 \\
15 & MYRTÁCEAE & Eucalyptus globulus & Eucalipto & 12 \\
16 & PINACEAE & Pinus patula & Pino & 10 \\
17 & MYRICACEAE & Morella pubescens & Laurel de cera & 10 \\
& TOTAL & & & 183 \\
\hline
\end{tabular}

Fuente: Rubiano (2019).

Al examinar el inventario e identificar cuáles son especies nativas y cuales especies introducidas, se determinó que de los 17 especies identificados en el inventario, 11 son especies nativas, lo que lleva a determinar que el $68 \%$ de la población total son especies nativas, que significa son mayoría, en contraste con el $38 \%$ que corresponde a las especies introducidas, aunque estas especies no presentan masas forestales constantes ya que no están dispersas por la cuenca media (Figura 2). A partir de los datos de abundancia y sus patrones de distribución por parches y en individuos aislados, se pudo analizar una pérdida de la biodiversidad producto de la degradación ambiental, que se ve reflejada en aspectos como el cambio en el paisaje, fisionomía de la vegetación y ausencia arbóreo dominante. Indicadores de la degradación ambiental, que ha sufrido la zona a lo largo del tiempo. 

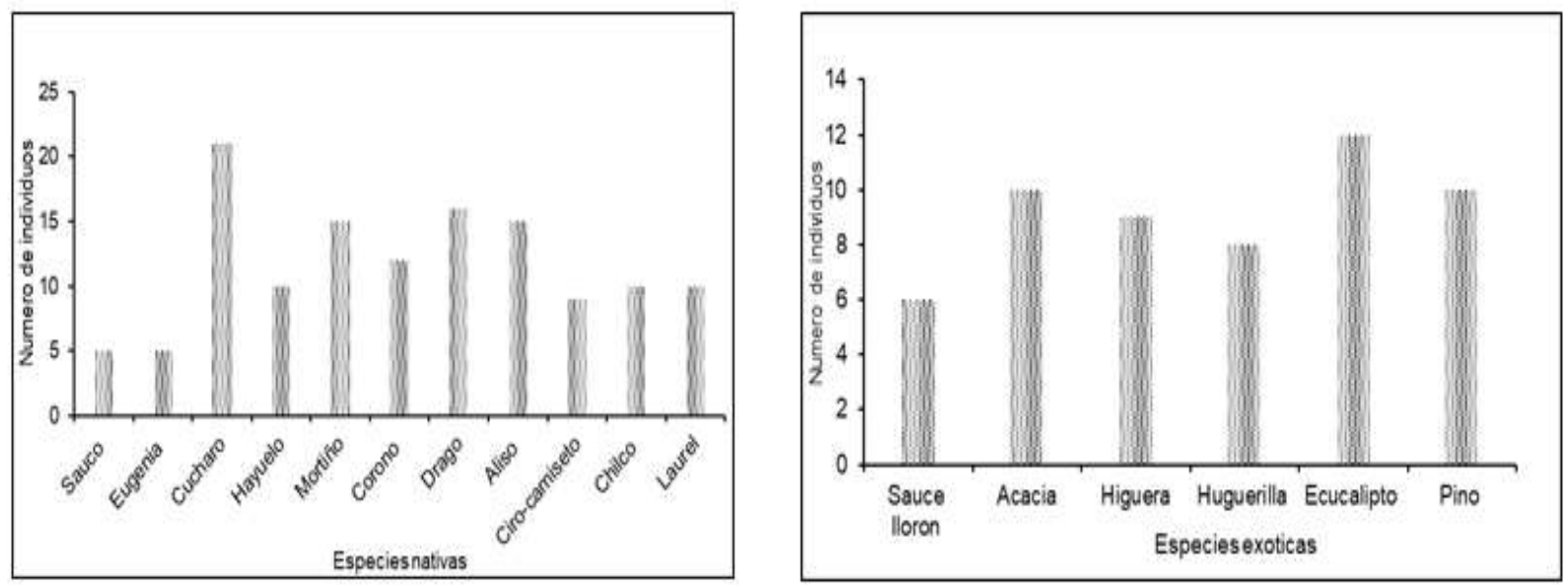

Figura 1. Especies nativas y exóticas, quebrada la Grande. Fuente: Camacho (2020).

Al revisar el DAP se encuentra una relación directa con la altura, donde las especies con mayor DAP fueron el Pino y la Acacia que además registran muy poca competencia. Sin embargo, el estrato dominante fueron los arbustos y árboles pequeños (Figura 2), todo aparentemente ocasionado por las acciones antrópicas que se observaron en la zona, así como la presencia de factores climáticos extremos que involucran la disminución en las precipitaciones anuales y mayor duración de meses secos, que de alguna manera indican procesos sucecionales debidos a la deforestación y perdida de árboles originales de la zona.

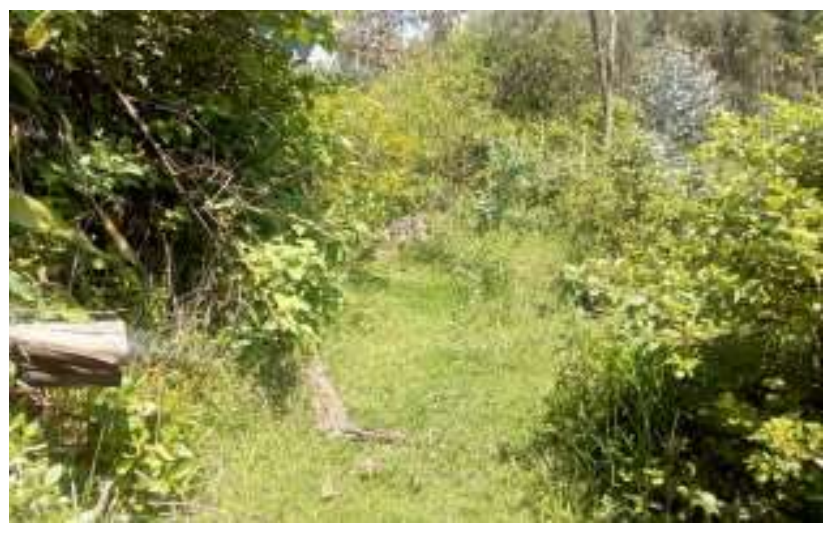

Figura 2. Relicto de Bosque Nativo. Fuente: Rubiano (2019).

Con respecto a la abundancia de las especies, los géneros con mayor número de plantas fueron el Cucharo (Myrsine guianensis) con 21 individuos, Drago (Croton sp) con 16 individuos, Mortiño (Vaccinium meridionale) con 15 individuos, seguido de Aliso (Alnus acuminata) con 15 individuos. (Figura 2) 


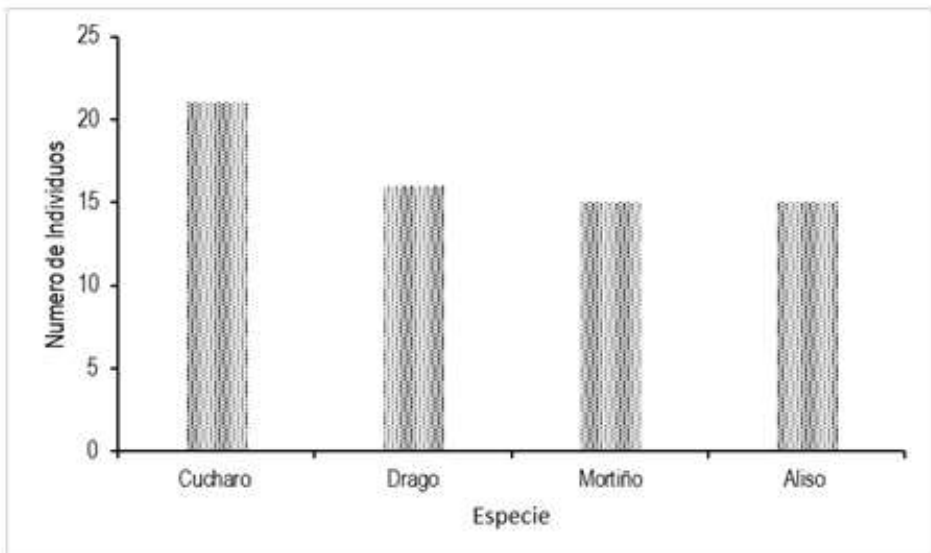

Figura 3. Especies con mayor abundancia en el Inventario. Fuente: Rubiano (2019).

Al examinar el inventario e identificar cuáles son especies nativas y cuales especies introducidas, se determinó que de los 17 especies identificados en el inventario, 11 son especies nativas, las cuales corresponden a Sauco (Sambucus nigra), Eugenia (Eugenia myrtifolia), Cucharo (Myrsine guianensis), Hayuelo (Dodonaea viscosa), Mortiño (Vaccinium meridionale), Corono, tabe (Xilosma spiculeferum), Drago (Croton sp), Aliso (Alnus acuminata), Ciro-camiseto (Baccharis macranta), Chilco (Baccharis latifolia), Laurel de cera (Morella pubescens) y por el otro lado los 6 géneros restantes son especies introducidas como Salix babylonica (Sauce llorón), Acacia (Acacia melanoxylon), Higuera (Ficus carica), Higuerilla (Ricinus communis), Eucalipto (Eucalyptus globulus), Pino (Pinus patula).

A partir de la observación se detecta la presencia de ciertos parches con individuos aislados, que sería un indicativo a tener en cuenta frente a posibles pérdidas de la biodiversidad producto de la degradación ambiental, que se ve reflejada en aspectos como el cambio en el paisaje (Figura 4), que evidencia acciones antrópicas como el pastoreo y la deforestación. Que se detecta gracias a indicadores ecológicos, como la presencia de especies Drago (Croton $\mathrm{sp})$, caracterizada por ser una indicadora en procesos de regeneración natural en zonas intervenidas, debido a las adaptaciones fisiológicas y estructurales que presenta como son la presencia de hojas compuestas, foliolos pequeños, presencia de espinas o aguijones, hábitos de crecimiento y formas de vida. Así mismo no hay un estrato arbóreo dominante que ratifica la pérdida de biodiversidad, aun mas cuando se observaron grupos de especies solitarias, lo que confirmo la intervención que ha sufrido la zona a lo largo del tiempo. 


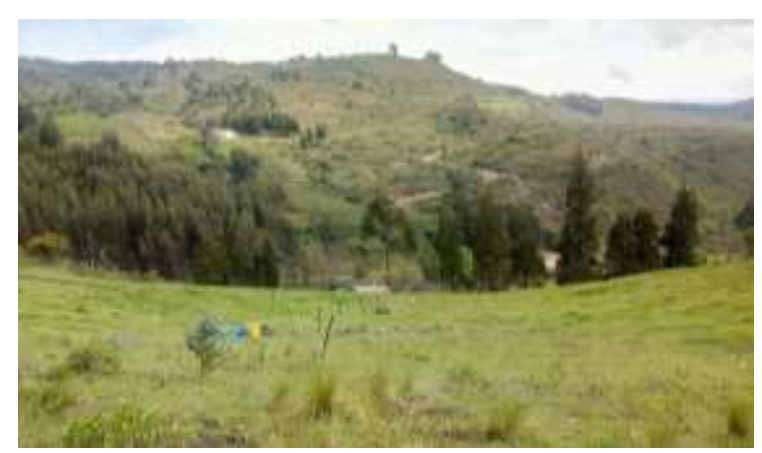

Figura 4. Área Deforestada. Sustitución de vegetación nativa por pastizales. Fuente: Rubiano (2019).

Producto de la investigación fue posible la identificación de los usos más comunes aplicados a las especies encontradas en la zona de estudio (anexos 1), lo que permitido identificar la posibilidad de implementar una estrategia de producción sostenible mediante el establecimiento de un sistema silvopastoril apoyado en especies como sauco y aliso. Asi mismo se propuso el desarrollo de una estrategia de conservación apoyada en el uso de especies como Aliso, Sauce y Corono que pudieran incidir positivamente en la mitigación de procesos erosivos identificados.

\section{CONCLUSIONES}

La microcuenca y su ecosistema presentan recursos naturales referentes a su flora que no han sido identificados o inventariados por lo que no se ha generado el desarrollo de propuestas de conservación o recuperación acordes con las necesidades de la zona.

Con el inventario se pudo determinar que la composición florística del área es un mosaico de relictos de bosque nativos, representado en especies arbustivas que hoy dominan el paisaje, combinados con especies introducidas.

Se observaron parches o relictos de bosque nativo, así como gran cantidad de especies arbustivas con caracteres xeromórficos como respuesta a procesos de adaptación producto de la disminución de las precipitaciones que se convierten en indicadores de procesos sesiónales debido a la deforestación y perdida de árboles originales de la zona.

Las actividades antrópicas observadas en la zona (ganadería, agricultura y construcción de casas), son en su mayoría para consumo y uso personal, presentan un modelo irregular de ocupación que evidencia su falta de planeación, las cuales afectan principalmente al ecosistema que los rodea.

El área de estudio es un claro ejemplo de cómo la ampliación de la frontera agrícola, la deforestación, el conflicto en el uso del suelo, deterioran los elementos estructurales de un 
ecosistema y disminuyen sus servicios, como se pueden evidenciar en la oferta del recurso hídrico el cual no es constante en la quebrada.

\section{CONTRIBUCIÓN DE LA AUTORÍA}

Primer autor: metodología, investigación, análisis de datos, conceptualización, escritura, borrador original. Segundo autor: análisis de datos, escritura, revisión y edición.

\section{AGRADECIMIENTOS}

Se agradece a la Universidad Nacional abierta y a distancia, por su apoyo en el desarrollo del proyecto, así mismo a la Alcaldía de Tibasosa por su disponibilidad en la consecución de información requerida para el desarrollo del proyecto.

\section{REFERENCIAS}

Abud-H, M., y Torres, A. (2016). Caracterización florística de un bosque altoandino en el parque Nacional Natural Puracé, Cauca, Colombia. Boletín CientífiCo Centro de Museos Museo de Historia natural, 20(1), 27-39.

Alcaldía Tibasosa. (2016). EOT Esquema de Ordenamiento Territorial. Tibasosa.

Alcaldía de Tibasosa. (2015-2019). Plan de desarrollo "Unidad y compromiso por Tibasosa". Tibasosa.

Catálogo virtual de flora del Valle de Aburrá. (2019). Recuperado de https://catalogofloravalleF.eia.edu.co/

Instituto Humboldt. (11 de septiembre de 2017). Biodiversidad colombiana: números para tener en cuenta [Boletín de Prensa]. Recuperado de http://www.humboldt.org.co/es/boletines-y-comunicados/item/1087-biodiversidadcolombiana-numero-tener-en-cuenta

FAO Organización de las naciones unidas para la alimentación y la agricultura. (2018). El aumento de la contaminación del suelo amenaza la inocuidad y la seguridad alimentaria. Recuperado de http://www.fao.org/sustainable-forestmanagement/toolbox/modules/forest-inventory/basic-knowledge/es/

FAO Organización de las naciones unidas para la alimentacion y la agricultura. (2020). Conjunto de herramientas para la gestion forestal sostenible. Recuperado de http://www.fao.org/news/story/es/item/40952/icode/

Ferreira, R. (2018). Manual de inventarios Forestales. Colombia. Bogotá.

Ledo, A., Condes, S., y Montes, F. (2012). Revisión de índices de distribución espacial usados en inventarios forestales y su aplicación en bosques tropicales. Revista Peruana de Biología, 19, 113-124. 
Mahecha, G. (2004). Vegetación del Territorio CAR. Bogotá, D.C: Corporación Autónoma Regional de Cundinamarca.

Romero, D. (2001). La Agroindustria de Veracruz ante la Globalización. Problemas y Perspectivas. (Tesis de Maestría) Universidad de Veracruzana. México. Recuperado de https://cdigital.uv.mx/bitstream/handle/1944/49298/RomeroCastilloDaniel.pdf?sequ ence $=1$ \&isAllowed $=y$

Universidad de Antioquia. (2020). Banco de objeto de aprendiza de la formación: Brevo Higuera- Ficus carica. Recuperado de http://aprendeenlinea.udea.edu.co/ova/?q=node/389 